\title{
THE DOMAINS OF NORMALITY OF HOLOMORPHIC SELF-MAPS OF $C^{*}$
}

\author{
Janina Kotus
}

\section{Introduction and results}

The iteration theory of Fatou and Julia applies to analytic maps $f: D \rightarrow D$ where the domain $D$ is contained in $\overline{\mathbf{C}}$, and introduces the sets $N(f)=\{z$ : $z \in D,\left(f^{n}\right)$ is a normal family in some neighbourhood of $\left.z\right\}$ and a Julia set $J(f)=D-N(f)$. In 1953, Rådström [10] showed that, to obtain interesting results, it is necessary to assume $f$ not to be a Moebius transformation and the complement of $D$ to consist of at most two points. One may assume that the complement of $D$ is $\emptyset,\{\infty\}$, or $\{0, \infty\}$, and with this normalisation there are essentially the following cases:

I. $D=\overline{\mathbf{C}}, f$ rational,

II. $D=\mathbf{C}, f$ entire, III. $D=\mathbf{C}^{*}=\{z: 0<|z|<\infty\}$.

In the third case there are four types of function $f$, depending on the behaviour at the points $0, \infty$ (see [1]):

a) $f(z)=k z^{n}, k \neq 0, n \in \mathbf{Z}-\{0, \pm 1\}$,

b) $f(z)=\exp (G(z)), G$ non-constant entire,

c) $f(z)=z^{-n} \exp (G(z)), G$ non-constant entire,

d) $f(z)=z^{m} \exp \left(F\left(z^{-1}\right)+G(z)\right), F, G$ non-constant entire, $m \in \mathbf{Z}$.

Note that (a) and (b) belong to cases I and II, respectively, and for $k \geq 2$ and $f$ of type (c) we have $f^{k}$ of type (d).

In this paper we consider the dynamics of functions of class III(d), denoted by $\mathcal{R}$. This class of functions was first discussed by Rådström [10] and next by Baker [1], Bhattacharyya [3], Keen [6, 7], Kotus [8] and Makienko [9].

For $f \in \mathcal{R}$, the Julia set $J(f)$ is a non-empty perfect subset of $\mathbf{C}^{*}$ and also completely invariant, i.e. $f(J(f))=f^{-1}(J(f))=J(f)$ and $J\left(f^{p}\right)=J(f)$ for $p \in \mathbf{N}$ (see [10]). The dynamics on $N(f)$ is better understood for rational than for entire functions or for functions of class $\mathcal{R}$. It is a consequence of Sullivan's theorem which states that every component is eventually periodic. This theorem is true only for a certain subclass of $\mathcal{R}$ :

Theorem A. Let $f \in \mathcal{R}$ have finitely many singular values. Then every component of $N(f)$ is eventually periodic. 
Theorem A was proved independently by Keen [6], Kotus [8] and Makienko [9]. A component $D$ of $N(f)$ is called parabolic at 0 (or at $\infty$ ) if $f(D) \subset D$ and $f^{n} \rightarrow 0$ in $D$ (or $f^{n} \rightarrow \infty$ in $D$ ).

In [8] the following theorem was proved:

Theorem B. Let $f \in \mathcal{R}$ have finitely many singular values. Then $f$ does not have a parabolic component at 0 and $\infty$.

A consequence of both theorems is a classification of periodic components. The aim of further investigation is a description of wandering components. The next theorem, proved by Baker [1], implies that any such component is simplyconnected for $n \geq n_{0}$.

Theorem C. If $f \in \mathcal{R}$, components of $N(f)$ are simply or doubly-connected. There is at most one doubly-connected component.

The known examples of wandering components were constructed by Baker [1] and belong to class III(b). Now we show the following examples.

Theorem 1. There exist functions $f_{1}, f_{2} \in \mathcal{R}$ such that:

a) $f_{1}$ has a wandering component $D$ of $N\left(f_{1}\right)$ such that the limit set of $f_{1}^{n}(D)$ equals one of the two essential singularities,

b) $f_{2}$ has a wandering component $D$ of $N\left(f_{2}\right)$ such that the limit set of $f_{2}^{n}(D)$ equals $\{0, \infty\}$.

Theorem 2. There is a function $f \in \mathcal{R}$ which has a wandering component $D$ of $N(f)$ with an infinite limit set.

The construction of these examples depends on the results obtained on complex approximation. This method of construction of components for entire functions was introduced by A. Eremenko and M.Yu. Lyubich [4] and improved by I.N. Baker [2]. We modify their constructions to obtain analogous examples of wandering components for functions of class $\mathcal{R}$. Also applying the results on complex approximation, we give examples of parabolic domains.

Theorem 3. There are functions of class $\mathcal{R}$ admitting parabolic domains at 0 or at $\infty$.

\section{Preliminary lemmas}

We shall make use of the following results.

Lemma 1 (Runge, see e.g. [5]). Suppose that $K$ is compact in $\mathbf{C}$ and $f$ is holomorphic on $K$; let also $\varepsilon>0$. Let $E$ be a set such that $E$ meets every component of $\overline{\mathbf{C}}-K$. Then there exists a rational function $r$ with poles in $E$ such that

$$
|f(z)-r(z)|<\varepsilon, \quad z \in K \text {. }
$$


Lemma 2. ([5], p. 131) Suppose that $E$ is a closed set in $\mathbf{C}$ and $f$ is a function defined on $E$. Then $f$ can be uniformly approximated on $E$ by meromorphic functions without poles in $E$ if and only if $f$ can be uniformly approximated by rational functions on each compact subset of $E$.

Lemma 3 ([5], p. 137). Suppose that $E$ is a closed set in $\mathbf{C}$ and that $z_{1}, z_{2}$ lie in the same component of $\mathbf{C}-E$. Then for each function $m$ meromorphic in $\mathbf{C}$ with a pole at $z_{1}$ and for each $\varepsilon>0$ there exists a function $m^{*}$ meromorphic in $\mathbf{C}$ which is analytic at $z_{1}$, has a pole at $z_{2}$, has no other poles except those of $m$, and for which

$$
\left|m(z)-m^{*}(z)\right|<\varepsilon, \quad z \in E .
$$

Lemma 4 ([5], p. 140). Suppose that $E$ is a closed set in $\mathbf{C}$ such that (i) $\overline{\mathbf{C}} \backslash E$ is locally connected at $\infty$.

If the meromorphic function $m$ has no poles on $E$, then for each $\varepsilon>0$ there exist a rational function $r$ with poles outside $E$ and an entire function $g$ such that $|m(z)-(r+g)(z)|<\varepsilon, z \in E$.

Remark 1. Let $m$ be a meromorphic function such that $m(z)=w\left(z^{-1}\right)+$ $g(z)$, where $w$ is a polynomial, while $g$ is entire. Then the function $f(z)=$ $\exp \left(w\left(z^{-1}\right)+g(z)\right)$ belongs to $\mathcal{R}$.

The next lemma is based on the main lemma proved in [4], p. 460.

Lemma 5. Let $K_{n}, L_{n}, n=1,2, \ldots$, be compact subsets of $\mathbf{C}^{*}$ with the following properties:

(i) $K_{n}, L_{n}$ are simply-connected for every $n$;

(ii) $K_{n} \cap K_{m}=\emptyset$ and $L_{n} \cap L_{m}=\emptyset$ for $n \neq m$;

(iii) $\left(\bigcup_{n=1}^{\infty} K_{n}\right) \cap\left(\bigcup_{n=1}^{\infty} L_{n}\right)=\emptyset$;

(iv) $\max \left\{|z|: z \in K_{n}\right\} \rightarrow 0, \min \left\{|z|: z \in L_{n}\right\} \rightarrow \infty$ as $n \rightarrow \infty$ and $2 \max \{|z|$ : $\left.z \in K_{n}\right\}<\frac{1}{2} \min \left\{|z|: z \in L_{n}\right\}$.

Let $w_{n} \in K_{n}, z_{n} \in L_{n}, \varepsilon_{n}>0$ and the function $h$ be analytic on $\cup_{n=1}^{\infty} K_{n} \cup$ $\cup_{n=1}^{\infty} L_{n}$. Then there exist non-constant entire functions $F, G$ such that $g(z)=$ $\exp \left(F\left(z^{-1}\right)+G(z)\right)$ satisfies

$$
\begin{gathered}
|g(z)-h(z)|<\varepsilon, \quad z \in K_{n} \cup L_{n}, \\
g\left(w_{n}\right)=h\left(w_{n}\right), \quad g\left(z_{n}\right)=h\left(z_{n}\right), \\
g^{\prime}\left(w_{n}\right)=h^{\prime}\left(w_{n}\right), \quad g^{\prime}\left(z_{n}\right)=h^{\prime}\left(z_{n}\right), \quad n=1,2, \ldots .
\end{gathered}
$$

In the proof we apply the following lemma. 
Lemma 6 ([4], p. 460). Let $A$ be a locally convex topological space, $V$ a domain in $A, W$ a convex subset in $V$ and $S$ an affine subspace of $A$ of finite codimension, such that $S \cap V \neq \emptyset$. Then $S \cap W$ is dense in $S \cap V$.

Proof of Lemma 2. Let $U$ be a union of two simply-connected sets which are neighbourhoods of $K_{1}$ and $L_{1}$ such that $h$ is analytic in $U$ and $U \cap K_{n}=\emptyset=$ $U \cap L_{n}=\emptyset$ for $n \geq 2$. Consider the space $A$ of all functions analytic in $U$ with the topology of uniform convergence on compact sets. Then

$$
V=\left\{g:|g(z)-h(z)|<\frac{1}{2} \varepsilon_{1}, z \in K_{1} \cup L_{1}\right\}
$$

is a convex domain in $A$. Let $W$ be the subset of rational functions with poles at 0 and at $\infty$ only. By Lemma $1, W$ is dense in $V$. Clearly $W$ is also convex. We also consider the affine subspace

$$
\begin{array}{rlrl}
S=\left\{g \in A: g\left(w_{1}\right)\right. & =h\left(w_{1}\right), & & g\left(z_{1}\right)=h\left(z_{1}\right), \\
g^{\prime}\left(w_{1}\right) & =h^{\prime}\left(w_{1}\right), & \left.g^{\prime}\left(z_{1}\right)=h^{\prime}\left(z_{1}\right)\right\} .
\end{array}
$$

By Lemma 6 there exists a rational function $g_{1} \in W \cap S$ such that $g_{1}(z)=$ $F_{1}\left(z^{-1}\right)+G_{1}(z)$, where $F_{1}, G_{1}$ are polynomials and

$$
\begin{array}{lrl}
\left|g_{1}(z)-h(z)\right|<\frac{1}{2} \varepsilon_{1}, & z \in K_{1} \cup L_{1}, \\
g_{1}\left(w_{1}\right)=h\left(w_{1}\right), & g_{1}\left(z_{1}\right)=h\left(w_{1}\right), \\
g_{1}^{\prime}\left(w_{1}\right)=h\left(w_{1}\right), & g_{1}^{\prime}\left(z_{1}\right)=h^{\prime}\left(z_{1}\right) .
\end{array}
$$

For $n>1$ there is a rational function $g_{n}$ such that $g_{n}(z)=F_{n}\left(z^{-1}\right)+G_{n}(z)$, where $F_{n}, G_{n}$ are polynomials and

$$
\begin{gathered}
\left|\sum_{k=1}^{n} g_{k}(z)-h(z)\right|<\frac{1}{2} \varepsilon_{n}, \quad z \in K_{n} \cup L_{n}, \\
\left|g_{k}(z)\right|<2^{-n+k} \varepsilon_{k}, \quad z \in K_{k} \cup L_{k} \quad \text { and } k<n, \\
\left|g_{n}(z)\right|<2^{-n} 2 \max \left\{|\zeta|: \zeta \in K_{n}\right\}<|z|<\frac{1}{2} \min \left\{|\zeta|: \zeta \in L_{n}\right\} \\
\sum_{k=1}^{n} g_{k}\left(w_{i}\right)=h\left(w_{i}\right), \quad \sum_{k=1}^{n} g_{k}\left(z_{i}\right)=h\left(z_{i}\right), \quad 1 \leq i \leq n, \\
\sum_{k=1}^{n} g_{k}^{\prime}\left(w_{i}\right)=h^{\prime}\left(w_{i}\right), \quad \sum_{k=1}^{n} g_{k}^{\prime}\left(z_{i}\right)=h\left(z_{i}\right), \quad 1 \leq i \leq n .
\end{gathered}
$$

It follows from (6) that the series $g=\sum_{n=1}^{\infty} g_{n}$ converges uniformly on the compact subsets of $\mathbf{C}^{*}$ and defines a function $g: \mathbf{C}^{*} \rightarrow \mathbf{C}$ of the form $g(z)=$ $F\left(z^{-1}\right)+G(z)$. Next, (4) and (5) imply (1), while (7) and (8) imply (2) and (3).

Remark 2. Let $g$ be the function of the form $g(z)=F\left(z^{-1}\right)+G(z)$, with $F, G$ non-constant entire. Then $f=\exp g$ belongs to $\mathcal{R}$. 
Lemma 7 ([4], p. 461). Let $f(z)=z+g(z)$ be an analytic function in the $\operatorname{disk}\{z:|z|<R\}$ such that $g(0)=g^{\prime}(0)=0$ and $|g(z)|<\varepsilon R$ and some $\varepsilon<\frac{1}{2}$. Then

$$
\begin{aligned}
& |z|\left(1-\frac{\varepsilon}{R}|z|\right) \leq|f(z)| \leq|z|\left(1+\frac{\varepsilon}{R}|z|\right), \\
& |\arg f(z)-\arg z|<2 \frac{\varepsilon}{R}|z|, \quad|z|<R .
\end{aligned}
$$

Lemma 8 ([4], p. 461). Let $q>1$. Then there exists a number $s(q)$ such that the estimates

$$
\begin{aligned}
& s_{0} \sum_{k=0}^{n-1} \varepsilon_{k}<s(q), \quad s_{0}>0, \quad \varepsilon_{k}>0, \\
& s_{k}\left(1-\varepsilon_{k} s_{k}\right) \leq s_{k+1} \leq s_{k}\left(1+\varepsilon_{k} s_{k}\right), \quad 0 \leq k \leq n-1,
\end{aligned}
$$

imply that

$$
\frac{1}{q} s_{0} \leq s_{k} \leq q s_{0}, \quad 0 \leq k \leq n .
$$

Lemma 9. If $f \in \mathcal{R}$ and $D_{i}, i=1,2$, are components of $N(f)$ such that $f^{p}\left(D_{i}\right) \subset D_{i}$ for some $p \in \mathbf{N}$ and $f^{n p} \rightarrow 0$ in $D_{1}, f^{n p} \rightarrow \infty$ in $D_{2}$. Then there are $f^{p}$ invariant curves $\gamma_{i}$ and positive constants $a_{i}, b_{i}$ such that $\gamma_{1}$ tends to 0 in $D_{1}, \gamma_{2}$ to $\infty$ in $D_{2}$ and $a_{i}|z|<\left|f^{p}(z)\right|<b_{i}|z|, z \in \gamma_{i}$.

The proof of this lemma is analogous to the proof of Theorem 2 in [2], p. 503.

\section{Proof of Theorem 3}

Denote

$$
\begin{gathered}
A=\left\{z:|z+2|<\frac{1}{2}\right\}, \\
A^{\prime}=\{z:|z+2|<1\}, \\
B=\left\{z: 10<|z| \text { and } 0<\arg z<\frac{1}{2} \pi\right\}, \\
B^{\prime}=\left\{z: 9<|z| \text { and }-0.1<\arg z<\frac{1}{2} \pi+0.1\right\}, \\
D=\left\{z: 0<|z|<0.1 \text { and }-\frac{1}{2} \pi<\arg z<0\right\}, \\
D^{\prime}=\left\{z: 0<|z|<0.2 \text { and }-\left(\frac{1}{2} \pi+0.1\right)<\arg z<0.1\right\} .
\end{gathered}
$$

Define functions $g$ and $h$ on the set $A^{\prime} \cup B \cup \partial B^{\prime}$ by

$$
g(z)= \begin{cases}\log 2+i \pi & \text { if } z \in A^{\prime} \cup \partial B^{\prime} \\ \log \left(10|z|^{1 / 2}\right)+i\left(\frac{1}{2} \arg z+\frac{1}{6} \pi\right) & \text { if } z \in B\end{cases}
$$




$$
h(z)= \begin{cases}\log 2+i \pi & \text { if } z \in A^{\prime} \cup \partial B^{\prime} \\ -\log \left(100|z|^{1 / 2}\right)-i\left(\frac{1}{2} \arg z+\frac{1}{6} \pi\right) & \text { if } z \in B .\end{cases}
$$

Then the functions $\exp g$ and $\exp h$ have the form

$$
\begin{gathered}
\exp g(z)= \begin{cases}-2 & \text { if } z \in A \cup \partial B^{\prime} \\
10 z^{1 / 2} e^{i \pi / 6} & \text { if } z \in B,\end{cases} \\
\exp h(z)= \begin{cases}-2 & \text { if } z \in A \cup \partial B^{\prime} \\
100^{-1} z^{-1 / 2} e^{-i \pi / 6} & \text { if } z \in B,\end{cases}
\end{gathered}
$$

where $z^{1 / 2}$ means one branch of the root function.

Clearly $\exp g(B) \subset B, \exp g\left(A^{\prime}\right) \subset A, \exp h\left(A^{\prime}\right) \subset A, \exp h(B) \subset D$. Let $C=\bar{A}^{\prime} \cup \bar{B} \cup \partial B^{\prime}$. Then $C$ and $g, h$ satisfy the assumptions of Lemma 2, so there are meromorphic functions $m_{1}, m_{2}$ with no poles on $C$ such that

$$
\begin{aligned}
& \left|m_{1}(z)-g(z)\right|<\frac{1}{2} \delta, \quad z \in C, \\
& \left|m_{2}(z)-h(z)\right|<\frac{1}{2} \delta, \quad z \in C,
\end{aligned}
$$

where $\delta$ is a small positive number. Since $C$ also satisfies the assumptions of Lemma 4 , there exist rational functions $r_{1}, r_{2}$ with poles outside $C$, and entire functions $e_{1}, e_{2}$ such that

$$
\begin{aligned}
& \left|m_{1}(z)-\left(r_{1}(z)+e_{1}(z)\right)\right|<\frac{1}{2} \delta, \quad z \in C, \\
& \left|m_{2}(z)-\left(r_{2}(z)+e_{2}(z)\right)\right|<\frac{1}{2} \delta, \quad z \in C .
\end{aligned}
$$

Applying Lemma 3 we can choose $r_{1}$ such that it has exactly one pole in $\mathbf{C}-C$ at say $a=0$. We can suppose that $r_{1}$ really has a pole at $a$ since the addition of $\lambda_{1} / z$, where $\lambda_{1}$ is a sufficiently small constant, will bring this about without spoiling the approximation properties listed above. Thus we may assume that $m_{1}(z)=\lambda_{1} z^{-1}+e_{1}(z)$ and $m_{2}(z)=\lambda_{2} z^{-1}+e_{2}(z)$. By Remark 1, $f_{1}=\exp m_{1}$ and $f_{2}=\exp m_{2}$ belong to the class $\mathcal{R}$. Now, we show

$$
\delta=\log (1+\mu)+i \varepsilon
$$

with $0<\mu<0.1,0<\varepsilon<0.1, f_{1}(B) \subset B, f_{2}(B) \subset D$. By (13-16) and (17) we have

$$
\operatorname{Re} m_{1}(z)>\operatorname{Re} g(z)-\log (1+\mu)=\log \left(10|z|^{1 / 2}(1+\mu)^{-1}\right)>\log 10
$$


and

$\operatorname{Re} m_{2}(z)<\operatorname{Re} h(z)+\log (1+\mu)=\log \left(100^{-1}|z|^{-1 / 2}(1+\mu)^{-1}\right)>\log 10^{-1}$.

As $w(z)=z^{-1}$ maps $D$ onto $B$ for $f_{3}=f_{2} \circ w$, we have $f_{3}(D) \subset D$ and $f_{3} \in \mathcal{R}$.

Next, (11), (12) and (17) imply

$$
\begin{gathered}
\left|f_{1}(z)\right|>\left|\exp m_{1}(z)\right|(1+\mu)>2|z|, \quad z \in B \\
\left|f_{3}(z)\right|<\left|\exp m_{2}\left(z^{-1}\right)\right|(1+\mu)^{-1}<\frac{1}{2}|z|, \quad z \in D .
\end{gathered}
$$

Hence all orbits of $f_{1}$ in $B$ tend to $\infty$ and all orbits of $f_{3}$ tend to 0 in $D$. Thus $B \subset N\left(f_{1}\right), D \subset N\left(f_{3}\right)$. The functions $f_{i}, i=1$ or $3 \operatorname{map} A^{\prime}$ into $A$, so $A^{\prime}$ contains attractive fixed points $\zeta_{i}, f_{i}^{n} \rightarrow \zeta_{i}$ in $A^{\prime}$ and $A^{\prime} \subset N\left(f_{i}\right)$. Further, $f_{1}\left(\partial B^{\prime}\right) \subset A^{\prime}, f_{3}\left(\partial D^{\prime}\right) \subset A^{\prime}, B$ is contained in a parabolic component of $N\left(f_{1}\right)$ at $\infty$ and $D$ in a parabolic component of $N\left(f_{3}\right)$ at 0 .

\section{Proof of Theorem 1}

We first construct an example of a function $f \in \mathcal{R}$ which has wandering components $D_{k}$ of $N(f)$ such that $f^{n} \rightarrow \infty$ in $D_{k}, k, n \in \mathbf{N}$.

Define $\varepsilon_{n}=10^{-n-3}, n \in \mathbf{N}$, and $\eta_{1}=0, \eta_{2}=\varepsilon_{1}, \eta_{n}=\sum_{k=1}^{n-1} 2^{-n+k+1} \varepsilon_{k}$ for $n>2$, so $\eta_{n+1}=\frac{1}{2} \eta_{n}+\varepsilon_{n}$. Write $c_{1}=2, c_{n}=2^{c-n-1}, n \in \mathbf{N}$ and, further, $a_{1}=1, b_{1}=2, a_{n}=\frac{2}{3} c_{n-1}$ and $b_{n}=c_{n-1}^{2}$ for $n>1$. Set

$$
\begin{gathered}
L_{n}=\left\{z: a_{n}<|z|<b_{n} \text { and }\left|\arg z-\frac{\pi}{2^{n}}\right|<\frac{1}{2^{n+3}}+\eta_{n}\right\}, \\
H_{n}=\left\{z: \ln a_{n}<\operatorname{Re} z<\ln b_{n} \text { and }\left|\operatorname{Im} z-\frac{\pi}{2^{n}}\right|<\frac{1}{2^{n+3}}+\eta_{n}\right\}, \quad n \in \mathbf{N} .
\end{gathered}
$$

Define the function $h$ on the set $\cup_{n=1}^{\infty} L_{n}$ by

$$
h(z)=\log \left(c_{n}|z|^{1 / 2}\right)+i \frac{1}{2} \arg z
$$

for $z \in L_{n}$. Then $\exp h(z)=c_{n} z^{1 / 2}, z \in L_{n}$, where $z^{1 / 2}$ means one branch of the root function. It is easy to check that $h\left(L_{n}\right) \subset H_{n+1}$, so $\exp h\left(L_{n}\right) \subset L_{n+1}$. The sets $L_{n}, n \in \mathbf{N}$, and the function $h$ satisfy the assumptions of Lemma 5 . Thus there is a function $g: \mathbf{C}^{*} \rightarrow \mathbf{C}, g(z)=F\left(z^{-1}\right)+G(z)$ where $F$ and $G$ are non-constant entire functions such that

$$
|g(z)-h(z)|<\delta_{n}, \quad z \in L_{n}
$$

here $\delta_{n}$ is a small positive number. Analogously the proof of Theorem 3 one can check that if

$$
\delta_{n}=\log \left(1+\mu_{n}\right)+i \varepsilon_{n}
$$


where $\mu_{n}>0$ and $\mu_{n} \rightarrow 0$, then $\exp g\left(L_{n}\right) \subset L_{n+1}$ for $n \in \mathbf{N}$. By (18) and (19) for $z \in L_{n}$,

$$
|\operatorname{Re} g(z)-\operatorname{Re} h(z)|<\log \left(1+\mu_{n}\right) .
$$

So

$$
\begin{aligned}
\operatorname{Re} g(z) & <\operatorname{Re} h(z)+\log \left(1+\mu_{n}\right)<\log \left(c_{n}|z|^{1 / 2}\left(1+\mu_{n}\right)\right) \\
& <\log c_{n}\left|c_{n-1}^{2}\right|^{1 / 2}\left(1+\mu_{n}\right)<\log c_{n}^{2}=\log b_{n+1}
\end{aligned}
$$

and

$$
\begin{aligned}
\operatorname{Re} g(z) & >\operatorname{Re} h(z)-\log \left(1+\mu_{n}\right)>\log \left(c_{n}|z|^{1 / 2}\left(1-\mu_{n}^{\prime}\right)\right) \\
& >\log c_{n}\left|\frac{2}{3} c_{n-1}\right|^{1 / 2}\left(1-\mu_{n}^{\prime}\right)>\log \frac{2}{3} c_{n}=\log a_{n+1} .
\end{aligned}
$$

Thus for $z \in L_{n}$

$$
a_{n+1}<|\exp g(z)|<b_{n+1} .
$$

Also by (18) and (19) we have

$$
\begin{aligned}
& \left|\operatorname{Im} g(z)-\frac{\pi}{2^{n+1}}\right|<\left|\operatorname{Im} h(z)-\frac{\pi}{2^{n+1}}\right|+\varepsilon_{n} \\
& \quad<\left|\frac{1}{2}\left(\frac{\pi}{2^{n}}+\frac{1}{2^{n+3}}+\eta_{n}\right)+\varepsilon_{n}-\frac{\pi}{2^{n+1}}\right|=\frac{1}{2^{n+4}}+\eta_{n+1} .
\end{aligned}
$$

Let $f=\exp g$. Then, by Remark $2, g$ belongs to $\mathcal{R}$. It follows from $(20)$ and (21) that $f\left(L_{n}\right) \subset L_{n+1}$ and so $f^{n} \rightarrow \infty$ uniformly in $L_{n}$. Thus $L_{n} \subset N(f)$. Let $N_{n}$ be a component of $N(f)$ containing $L_{n}$. Clearly $f: N_{n} \rightarrow N_{n+1}$. If some $N_{n}=N_{n+1}$, then $N_{n}$ is in fact an unbounded domain which is mapped into itself and in which $f^{n}(z) \rightarrow \infty$. By Lemma 9 there is a path $\gamma$ to $\infty$ in $N_{n}$ and positive constants $c, d$ such that $c|z|<|f(z)|<d|z|$ as $z \rightarrow \infty$ on $\gamma$. But this contradicts the growth implied by the construction on

$$
\left\{z:|z|=c_{n-1} \text { and }\left|\arg z-\frac{\pi}{2^{n}}\right|<\frac{1}{2^{n+3}}+\eta_{n}\right\} \subset N_{n} .
$$

We have $|f(z)|>a_{n+1}=\frac{2}{3} 2^{c_{n-1}}$. Thus all $N_{n}$ are different and each is a wandering component of $N(f)$. Analogously one can construct an example of wandering components in which $f^{n} \rightarrow 0$.

Now, we give an example of $f \in \mathcal{R}$ which has wandering components with limit set equals to $\{0, \infty\}$.

Let $L_{n}, H_{n}, \varepsilon_{n}, \eta_{n}$ be defined as above. Write $d_{n}=c_{n}^{-1}, r_{n}=b_{n}^{-1}$, $s_{n}=a_{n}^{-1}$ and set

$$
K_{n}=\left\{z: r_{n}<|z|<s_{n} \text { and }\left|\arg z+\frac{\pi}{2^{n}}\right|<\frac{1}{2^{n+3}}+\eta_{n}\right\}
$$




$$
D_{n}=\left\{z: \ln r_{n}<\operatorname{Re} z<\ln s_{n} \text { and }\left|\operatorname{Im} z+\frac{\pi}{2^{n}}\right|<\frac{1}{2^{n+3}}+\eta_{n}\right\}, \quad n \in \mathbf{N} .
$$

Define the function $h$ on the set $\cup_{n=1}^{\infty}\left(K_{n} \cup L_{n}\right)$ by

$$
h(z)= \begin{cases}-\left(\log d_{2 n}|z|^{1 / 2}+i \frac{1}{2} \arg x\right) & \text { if } z \in K_{2 n}, \\ -\left(\log c_{2 n-1}|z|^{1 / 2}+i \frac{1}{2} \arg x\right) & \text { if } z \in L_{2 n-1}, n \in \mathbf{N} .\end{cases}
$$

Clearly $h\left(L_{2 n-1}\right) \subset D_{2 n}, h\left(K_{2 n}\right) \subset H_{2 n+1}$. By Lemma 5 there is a function $g(z)=F\left(z^{-1}\right)+G(z), F$ and $G$ non-constant entire functions, satisfying

$$
\begin{gathered}
|g(z)-h(z)|<\delta_{2 n-1}, \quad z \in L_{2 n-1}, \\
|g(z)-h(z)|<\delta_{2 n}, \quad z \in K_{2 n}, n \in \mathbf{N},
\end{gathered}
$$

where $\delta_{2 n-1}$ and $\delta_{2 n}$ are so chosen that $f=\exp g$ maps $L_{2 n-1}$ into $K_{2 n}$ and $K_{2 n}$ into $L_{2 n+1}$. Hence $f^{2 n} \rightarrow \infty$ and $f^{2 n-1} \rightarrow 0$ uniformly in each $L_{2 n-1}$, and conversely $f^{2 n-1} \rightarrow \infty, f^{2 n} \rightarrow 0$ in each $K_{2 n}$. Let $N_{2 n-1}$ and $N_{2 n}$ be components of $N(f)$ containing $L_{2 n-1}$ and $K_{2 n}$, respectively. On

$$
\left\{z:|z|=d_{2 n-1} \text { and }\left|\arg z+\frac{\pi}{2^{2 n}}\right|<\frac{1}{2^{2 n+3}}+\eta_{2 n}\right\} \subset N_{2 n}
$$

we have

$$
\left|f^{2}(z)\right|<S_{2 n+2}=\frac{3}{2}\left(2^{c_{2 n}}\right)^{-1}=\frac{3}{2} 2^{-d_{2 n}}
$$

while on

$$
\left\{z:|z|=c_{2 n-2} \text { and }\left|\arg z-\frac{\pi}{2^{2 n-1}}\right|<\frac{1}{2^{2 n+2}}+\eta_{2 n-1}\right\} \subset N_{2 n-1}
$$

we have

$$
\left|f^{2}(z)\right|>a_{2 n+1}=\left(\frac{2}{3} 2^{c_{2 n}}\right) .
$$

This and Lemma 9 imply that all $N_{2 n-1}$ and $N_{2 n}$ are different components of $N(f)$.

\section{Proof of Theorem 2}

To construct this example, first fix $\varepsilon$ and $q$ such that $0<\varepsilon<\frac{1}{2}, 1<q<2^{1 / 3}$. Consider a sequence $r_{n}, 0 \leq n<\infty$, such that $0<r_{n}<\frac{1}{2} r_{n-1}, n=1,2, \ldots$, and

$$
\varepsilon \sum_{k=0}^{n-1} \frac{r_{n}}{r_{k}}<\min \left\{s, \frac{\pi}{8}\right\}
$$


where $s=s(q)$ is chosen to satisfy Lemma 8. Define a sequence $a_{0}=2, a_{2 k-1}=$ $4^{k}, a_{2 k}=4^{-k}, k \geq 1$. Let

$$
\begin{aligned}
& B_{n}=\left\{z:\left|z-a_{n}\right|<\frac{1}{2} r_{n}\right\}, \\
& D_{n}=\left\{z: q^{-2} r_{n}<|z|<q^{-1} r_{n},|\arg z|<\frac{1}{4} \pi\right\}, \\
& Q_{n}=\left\{z: q^{-3} r_{n}<\left|z-a_{n}\right|<r_{n},|\arg z|<\frac{1}{4} \pi\right\}, \quad n \geq 0,
\end{aligned}
$$

and define the function $h$ on the set $\cup_{n=0}^{\infty}\left(B_{n} \cup Q_{n}\right)$ by

$$
h(z)= \begin{cases}\log \left(z+a_{n+1}-a_{n}\right) & \text { if } z \in B_{n}, n \geq 0 \\ \log \left(q^{-3 / 2} r_{n+1}\right) & \text { if } z \in Q_{n}, n \geq 0 .\end{cases}
$$

See Figure 1.
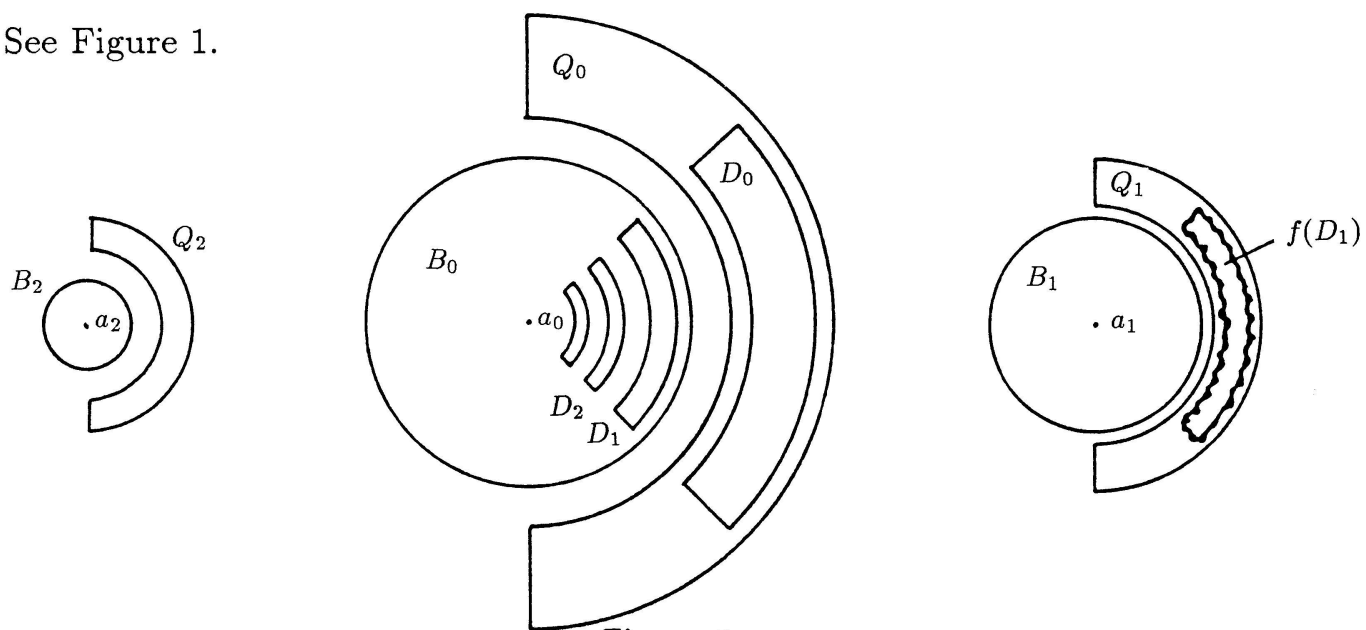

Figure 1.

By Lemma 5 there are non-constant entire functions $F$ and $G$ such that $f(z)=\exp \left(f\left(z^{-1}\right)+G(z)\right)$ satisfies

$$
\begin{gathered}
f\left(a_{n}\right)=a_{n+1}, \quad f^{\prime}\left(a_{n}\right)=1, \quad n \geq 0, \\
|f(z)-h(z)|<\frac{1}{4} \varepsilon r_{n}, \quad z \in B_{n},
\end{gathered}
$$

and

$$
f\left(Q_{n}\right) \subset D_{n+1} .
$$

Let $|z|<\frac{1}{2} r_{n}, s_{k}=\left|f^{k}(z)-a_{k}\right|, k \geq 0$. We shall prove that

$$
q^{-1} s_{0} \leq s_{k} \leq q s_{0}, \quad 0 \leq i \leq k-1<n .
$$


As $q s_{0}<\left(\frac{1}{2} 2^{1 / 3}\right) r_{n}<r_{n}<\frac{1}{2} r_{i}$, it follows that $f^{i}(z) \in B_{i}, 0 \leq i \leq k-1$. Then from (23) and (24) and Lemma 7 we have

$$
s_{i}\left(1-\varepsilon s_{i} / r_{i}\right) \leq s_{i+1} \leq s_{i}\left(1+\varepsilon s_{i} / r_{i}\right), \quad 0 \leq i \leq k-1 .
$$

Moreover,

$$
s_{0} \sum_{i=0}^{n-1} \frac{\varepsilon}{r_{i}} \leq \sum_{i=0}^{n-1} \frac{r_{n}}{r_{i}}<s .
$$

Using Lemma 8 we obtain $q^{-1} s_{0} \leq s_{k} \leq q s_{0}$. Thus (26) is proved by induction, and we have $f^{k}(z) \in B_{k}$ for $0 \leq k \leq n-1$.

Using Lemma 7 once more we obtain

$$
\left|\arg \left(f^{k+1} z-a_{k+1}\right)-\arg \left(f^{k} z-a_{k}\right)\right| \leq 2 \varepsilon \frac{s_{k}}{r_{k}} \leq 2 \varepsilon \frac{r_{n}}{r_{k}}, \quad 0 \leq k \leq n-1 .
$$

Therefore, by (22),

$$
\left|\arg \left(f^{n} z-a_{n}\right)-\arg z\right| \leq 2 \varepsilon \sum_{k=0}^{n-1} \frac{r_{n}}{r_{k}} \leq \frac{1}{4} \pi .
$$

It follows that (taking $k=n) f^{n}\left(D_{n}\right) \subset Q_{n}, n \geq 0$. Since $f\left(Q_{n}\right) \subset D_{n+1}$ by (25), it follows that every orbit originating from $D_{0}$ has a limit point at $a_{0}=2$, and, consequently, further limit points at $a_{1}=f\left(a_{0}\right), a_{2}, a_{3}, \ldots$ Moreover, it is clear from $f^{n+1}\left(D_{n}\right) \subset D_{n+1}$ that the limit functions are constants. As in the case of entire functions, the existence of an infinity of constant limit functions of $\left(f^{n}\right)$ in a component of $N(f)$ implies that the component is wandering. Thus the component which contains $D_{0}$ is wandering and the limit set of $f^{n} \mid D_{0}$ contains an oscillating trajectory $a_{0}, a_{1}, a_{2}, \ldots$

\section{References}

[1] BAKer, I.N.: Wandering domains for maps of the punctured plane. - Ann. Acad. Sci. Fenn. Ser. A I Math. 12, 191-198.

[2] BAKER, I.N.: Infinite limits in the iteration of entire functions. - Ergodic Theory Dynamical Systems 8, 1988, 503-507.

[3] Bhattacharyya, P.: Iteration of analytic functions. - Ph.D. thesis, University of London, 1969 (unpublished).

[4] Eremenko, A., and M.Yu. Lyubich: Examples of entire functions with pathological dynamics. - J. London Math. Soc. (2) 36, 1987, 458-468.

[5] Gaier, D.: Lectures on complex approximation. - Birkhäuser, Boston-Basel, 1985.

[6] KeEn, L.: Dynamics of holomorphic maps of $C^{*}$. - Proceedings of the Workshop on Holomorphic Functions and Moduli, Mathematical Sciences Research Institute, Berkeley, 1986. 
[7] KeEn, L.: Topology and growth of a special class of holomorphic self-maps of $C^{*}$. Ergodic Theory Dynamical Systems 9, 1989, 321-328.

[8] Kotus, J.: Iterated holomorphic maps on the punctured plane. - Preprint, Institute of Mathematics, Polish Academy of Sciences, Warsaw, 1986.

[9] Makienko, P.: Iterations of analytic functions in $C^{*}$. - Dokl. Akad. Nauk SSSR 297, 1987, 35-37 (Russian).

[10] RÅDSTRöM, H.: On the iteration of analytic functions. - Math. Scand. 1, 1953, 85-92.

Technical University of Warsaw

Institute of Mathematics

PL-00-061 Warsaw

Poland

Received 14 August 1989 